\title{
Crossability Studies of Interspecific Hybridization among Vigna Species
}

\author{
A Nishant Bhanu*, MN Singh and K Srivastava \\ Department of Genetics and Plant Breeding, Banaras Hindu University, India
}

Received: February 23, 2018; Published: March 05, 2018

*Corresponding author: A Nishant Bhanu, Department of Genetics and Plant Breeding, Institute of Agricultural Sciences, Banaras Hindu University, India, Email: nishant.bhanu@gmail.com

\begin{abstract}
A total of 80 interspecific crosses i.e., 36 each of $V$. radiata $\times V$. umbellata (V. radiata as female) and $V$. mungo $\times V$. umbellata (V. mungo as female) and 08 crosses of $V$. radiata $\times V$. mungo ( $V$. radiata as female) were attempted to study the crossability relationship among these three Vigna species. Among the crosses of V. radiata $\times$ V . umbellata the crossability was observed highest in HUM $12 \times$ RBL $9(16.27 \%)$ followed by HUM $12 \times$ RBL 9 (15.78\%). In case of $V$. mungo $\times$ V. umbellata, the maximum crossability of $11.36 \%$ was noticed in cross, Mash $338 \times$ RBL 9 . For $V$. radiata $\times V$. mungo, the highest crossability was visualized in hybrid, ML $1464 \times$ Mash 338 (37.5\%). The study indicated that different kinds of pre and post fertilization barriers are responsible for complete sterility to low fertility. RBL 1 and RBL 9 gnotypes of ricebean showing substantially high percent of crossability and better seed set with different cultivars of mungbean and blackgram may be utilized for genetic improvement of the mungbean and blackgram.
\end{abstract}

Keywords: Interspecific hybridization; Crossability; V. radiata; V. mungo; V. umbellata

\section{Introduction}

Legumes are next to cereals in terms of their economic and nutritional importance as human food. They also play an important role in maintaining soil fertility and sustainability of production systems. Among several pulses grown mungbean and blackgram are one of the important grain legumes grown throughout the year. Being a short duration crop they can be a better option for enhancing the pulse production of pulses. However, the total production and productivity of mungbean is affected by a number of biotic and abiotic factors. Among biotic factors, Mungbean Yellow Mosaic Virus (MYMV) transmitted through whitefly, i.e., Bemesia tabaci is a major constraint to the cultivation of grain legumes in India, particularly mungbean and blackgram. The weather parameters play a vital role in survival and multiplication of white fly and influence MYMV outbreak during monsoon season. Management of this disease is only possible by the way of reducing the whitefly population using insecticides which are ineffective under severe infestations making complete destruction due to virus. Therefore, development and use of virus resistant cultivars turns out to be the most effective and economical strategy against MYMV [1]. Basic reason for limited success had been due to the limited variability prevailed among the mungbean and blackgram genotypes used for hybridization in most of the studies.
Interspecific hybridization plays a significant role in alien gene introgression and is the probable option for transferring the desirable genes of qualitative and quantitative characters in mungbean and blackgram. Ricebean [V. umbellata (Thunb.) Ohwi and Ohashi], a long duration (90-120 days) minor legume which is genetically close to mungbean and blackgram (all three species $2 \mathrm{n}=2 \mathrm{x}=22$ ) possess resistance for MYMV, CLS, bruchids, and powdery mildew. Successful hybridization primarily depends on the intercrossing potential/ crossability of the parents involved as well as development of the hybrid embryos including fertility of the $F_{1}$ hybrids and their derivatives. In interspecific crosses of food legumes failure of interspecific hybridization due to embryo degeneration is common [2,3]. Interspecific hybridization among mungbean, blackgram and ricebean with varying degree of success has been reported. Keeping this in view, the present piece of investigation was initiated to study the crossability relationship among three Vigna species viz. $V$. radiata (mungbean), V. mungo (blackgram) and V. umbellata (ricebean).

\section{Materials and Methods}

For the present experiment, a total of six diverse genotypes/ varieties, each of mungbean viz. Pusa 0672, ML 1464, SML 1455, 
HUM 12, KM 2241, TM 96-2, ricebean namely RBL 1, RBL 6, RBL 9, RBL 33, RBL 140, RBL 141 and blackgram viz. Mash 338, Mash 114, Co 5, Palampur 93, Shekhar 2 and T 9 were selected. The experimental material was planted in crossing block in cemented pots at two different dates of sowing of 10 days intervals (August 10 and August 20, 2014) at Agricultural Research Farm, Banaras Hindu University Varanasi during Kharif season, 2014 [4-6]. Buds of optimum size of the female parent were emasculated the day before anthesis (1600 to $1800 \mathrm{HRS}$ ) and pollinated in the next morning (0600 to $0800 \mathrm{HRS}$ ). 8 to 12 flowers per plant per day were emasculated besides picking the self-pollinated flowers/ pods to avoid any severe load. Hybridization technique using hand emasculation and pollination was followed [7]. A total of 80 interspecific crosses i.e. 36 each of $V$. radiata $\times V$. umbellata (V. radiata as female) and $V$. mungo $\times V$. umbellata ( $V$. mungo as female) and 08 crosses of $V$. radiata $\times V$. mungo ( $V$. radiata as female) were accomplished. Observations were recorded on the number of buds emasculated, pollinated, pod initiated and matured pods harvested. Percent pod setting was obtained from [Number of pods set/ Number of buds pollinated] $\times 100$. Percent ovule fertility was calculated [Total No. of developed seed/ Total No. of ovule scar] $\times 100$. Meteorological observations were taken from the Meteorological Unit, Department of Agronomy, Institute of Agricultural Sciences, Banaras Hindu University, Varanasi.

\section{Results and Discussion}

Introgression of desirable gene into cultivated species could lead to development of high yielding varieties coupled with resistance for biotic and abiotic stresses. Close relatives of mungbean and blackgram have been used in the breeding programme. However, recovery of successful hybrid is difficult due to crossability barriers. In spite of these technical hitches, interspecific hybridization among Vigna speices has been successfully accomplished by many workers [8,9-12]. Further, in any interspecific hybridization, crossability is the pre-requisite for gene transfer. A better understanding of crossability relationship among the species had been helpful in opting methods for making successful crosses and also in drawing the phylogenetic relationship among species. V. umbellata possessing many desirable components coupled with resistance to MYMV, CLS and bruchids and powdery mildew can be useful in developing high yielding resistant varieties of mungbean and blackgram by transferring these genes into the cultivated species. The present investigation was carried out attempting interspecific hybridization with an objective to transfer useful traits from the $V$. mungo and V. umbellata into V. radiata and V. umbellata into $V$. mungo. The extent to crossability and ovule fertility was studied. The result of crosses pertaining to crossability and ovule fertility are furnished in (Table 1).

Table 1: Pod set, Crossability Percentage and Ovule Fertility Percentage among Vigna Species.

\begin{tabular}{|c|c|c|c|c|c|c|c|c|}
\hline Sl. No. & Cross & $\begin{array}{l}\text { No. of Buds } \\
\text { Emasculated } \\
\text { (1) }\end{array}$ & $\begin{array}{l}\text { No. of buds } \\
\text { pollinated } \\
\text { (2) }\end{array}$ & $\begin{array}{l}\text { No. of Buds } \\
\text { Fertilized (3) }\end{array}$ & $\begin{array}{l}\text { No. of Pods } \\
\text { Harvested } \\
\quad(4)\end{array}$ & $\begin{array}{l}\text { Crossability } \\
\text { Percentage } \\
(\%)[(2) /(4) \\
\times 100] \\
\end{array}$ & $\begin{array}{c}\text { Ovule } \\
\text { Fertility (\%) }\end{array}$ & Remarks \\
\hline \multicolumn{9}{|c|}{ Vigna Radiata $\times$ Vigna Umbellata } \\
\hline 1 & $\begin{array}{l}\text { TM 96-2 } \times \\
\text { RBL } 1\end{array}$ & 30 & 22 & 5 & 1 & 4.54 & 33.33 & $\begin{array}{l}\text { Viable Dimpled } \\
\text { Seeds }\end{array}$ \\
\hline 2 & $\begin{array}{l}\text { TM 96-2 × } \\
\text { RBL } 6\end{array}$ & 25 & 18 & 0 & 0 & 0.00 & 0 & $\begin{array}{c}\text { Absence of Seed set } \\
\text { and Abscission of } \\
\text { Crossed Flowers }\end{array}$ \\
\hline 3 & $\begin{array}{l}\text { TM } 96-2 \times \\
\text { RBL } 9\end{array}$ & 32 & 22 & 5 & 1 & 4.54 & 40.00 & $\begin{array}{l}\text { Viable Dimpled } \\
\text { Seeds }\end{array}$ \\
\hline 4 & $\begin{array}{l}\text { TM 96-2 } \times \\
\text { RBL } 33\end{array}$ & 75 & 60 & 4 & 2 & 3.33 & 42.85 & $\begin{array}{l}\text { Viable Dimpled } \\
\text { Seeds }\end{array}$ \\
\hline 5 & $\begin{array}{l}\text { TM 96-2 } \times \\
\text { RBL } 140\end{array}$ & 32 & 26 & 3 & 1 & 3.84 & 25.00 & $\begin{array}{l}\text { Tiny, Dimpled } \\
\text { Seeds }\end{array}$ \\
\hline 6 & $\begin{array}{l}\text { TM 96-2 × } \\
\text { RBL } 141\end{array}$ & 35 & 28 & 4 & 1 & 3.57 & 28.57 & $\begin{array}{l}\text { Tiny, Dimpled } \\
\text { Seeds }\end{array}$ \\
\hline 7 & $\begin{array}{l}\text { Pusa } 0672 \times \\
\text { RBL } 1\end{array}$ & 45 & 34 & 9 & 5 & 14.70 & 26.67 & $\begin{array}{l}\text { Viable Dimpled } \\
\text { Seeds }\end{array}$ \\
\hline 8 & $\begin{array}{l}\text { Pusa } 0672 \times \\
\text { RBL } 6\end{array}$ & 65 & 56 & 8 & 5 & 8.92 & 15.62 & $\begin{array}{l}\text { Viable Dimpled } \\
\text { Seeds }\end{array}$ \\
\hline 9 & $\begin{array}{l}\text { Pusa } 0672 \times \\
\text { RBL } 9\end{array}$ & 50 & 41 & 11 & 5 & 12.19 & 44.12 & $\begin{array}{l}\text { Tiny, Dimpled } \\
\text { Seeds }\end{array}$ \\
\hline 10 & $\begin{array}{c}\text { Pusa } 0672 \times \\
\text { RBL } 33\end{array}$ & 22 & 16 & 3 & 0 & 0.00 & 0 & $\begin{array}{l}\text { Abscission of Young } \\
\text { Fruits } \\
\end{array}$ \\
\hline 11 & $\begin{array}{l}\text { Pusa } 0672 \times \\
\text { RBL } 140\end{array}$ & 24 & 16 & 0 & 0 & 0.00 & 0 & $\begin{array}{l}\text { Absence of Seed Set } \\
\text { and Abscission of } \\
\text { Crossed Flowers }\end{array}$ \\
\hline 12 & $\begin{array}{l}\text { Pusa } 0672 \times \\
\text { RBL } 141\end{array}$ & 32 & 26 & 4 & 1 & 3.84 & 14.28 & $\begin{array}{l}\text { Viable Dimpled } \\
\text { Seeds }\end{array}$ \\
\hline
\end{tabular}




\begin{tabular}{|c|c|c|c|c|c|c|c|c|}
\hline 13 & $\begin{array}{l}\text { KM } 2241 \times \\
\quad \text { RBL } 1\end{array}$ & 22 & 18 & 3 & 0 & 0.00 & 0 & $\begin{array}{l}\text { Abscission of Young } \\
\text { Fruits }\end{array}$ \\
\hline 14 & $\begin{array}{l}\text { KM } 2241 \times \\
\quad \text { RBL } 6\end{array}$ & 24 & 18 & 0 & 0 & 0.00 & 0 & $\begin{array}{l}\text { Absence of Seed set } \\
\text { and Abscission of } \\
\text { Crossed Flowers }\end{array}$ \\
\hline 15 & $\begin{array}{l}\text { KM } 2241 \times \\
\text { RBL } 9\end{array}$ & 30 & 24 & 4 & 1 & 4.10 & 44.44 & $\begin{array}{l}\text { Viable Dimpled } \\
\text { Seeds }\end{array}$ \\
\hline 16 & $\begin{array}{l}\text { KM } 2241 \times \\
\text { RBL } 33\end{array}$ & 45 & 42 & 8 & 3 & 7.14 & 30.77 & $\begin{array}{l}\text { Viable Dimpled } \\
\text { Seeds }\end{array}$ \\
\hline 17 & $\begin{array}{l}\text { KM } 2241 \times \\
\text { RBL } 140\end{array}$ & 22 & 17 & 3 & 0 & 0.00 & 0 & $\begin{array}{l}\text { Abscission of Young } \\
\text { Fruits }\end{array}$ \\
\hline 18 & $\begin{array}{l}\text { KM } 2241 \times \\
\text { RBL } 141\end{array}$ & 40 & 35 & 5 & 1 & 2.85 & 36.37 & $\begin{array}{l}\text { Viable Dimpled } \\
\text { Seeds }\end{array}$ \\
\hline 19 & $\begin{array}{l}\text { ML } 1464 \times \\
\text { RBL } 1\end{array}$ & 45 & 42 & 9 & 4 & 9.52 & 64.70 & $\begin{array}{l}\text { Viable Dimpled } \\
\text { Seeds }\end{array}$ \\
\hline 20 & $\begin{array}{l}\text { ML } 1464 \times \\
\text { RBL } 6\end{array}$ & 45 & 42 & 9 & 5 & 11.90 & 47.05 & $\begin{array}{l}\text { Tiny, Dimpled } \\
\text { Seeds }\end{array}$ \\
\hline 21 & $\begin{array}{l}\text { ML } 1464 \times \\
\text { RBL } 9\end{array}$ & 42 & 36 & 8 & 4 & 11.11 & 55.55 & $\begin{array}{l}\text { Viable Dimpled } \\
\text { Seeds }\end{array}$ \\
\hline 22 & $\begin{array}{l}\text { ML } 1464 \times \\
\text { RBL } 33\end{array}$ & 45 & 38 & 6 & 3 & 7.89 & 20.00 & $\begin{array}{l}\text { Tiny, Dimpled } \\
\text { Seeds }\end{array}$ \\
\hline 23 & $\begin{array}{l}\text { ML } 1464 \times \\
\text { RBL } 140\end{array}$ & 18 & 15 & 0 & 0 & 0.00 & 0 & $\begin{array}{l}\text { Absence of Seed Set } \\
\text { and Abscission of } \\
\text { Crossed Flowers }\end{array}$ \\
\hline 24 & $\begin{array}{l}\text { ML } 1464 \times \\
\text { RBL } 141\end{array}$ & 20 & 16 & 0 & 0 & 0.00 & 0 & $\begin{array}{l}\text { Absence of Seed Set } \\
\text { and Abscission of } \\
\text { Crossed Flowers }\end{array}$ \\
\hline 25 & $\begin{array}{l}\text { HUM } 12 \times \\
\text { RBL } 1\end{array}$ & 56 & 43 & 12 & 7 & 16.27 & 70.00 & $\begin{array}{l}\text { Viable Dimpled } \\
\text { Seeds }\end{array}$ \\
\hline 26 & $\begin{array}{l}\text { HUM } 12 \times \\
\quad \text { RBL } 6\end{array}$ & 35 & 28 & 10 & 4 & 14.28 & 44.44 & $\begin{array}{l}\text { Viable Dimpled } \\
\text { Seeds }\end{array}$ \\
\hline 27 & $\begin{array}{l}\text { HUM } 12 \times \\
\text { RBL } 9\end{array}$ & 50 & 38 & 10 & 6 & 15.78 & 66.67 & $\begin{array}{l}\text { Viable Dimpled } \\
\text { Seeds }\end{array}$ \\
\hline 28 & $\begin{array}{c}\text { HUM } 12 \times \text { RBL } \\
33\end{array}$ & 35 & 24 & 7 & 2 & 8.33 & 40.00 & $\begin{array}{l}\text { Tiny, Dimpled } \\
\text { Seeds }\end{array}$ \\
\hline 29 & $\begin{array}{c}\text { HUM } 12 \times \text { RBL } \\
140\end{array}$ & 30 & 20 & 1 & 0 & 0.00 & 0 & $\begin{array}{c}\text { Abscission of Young } \\
\text { Fruits }\end{array}$ \\
\hline 30 & $\begin{array}{c}\text { HUM } 12 \times \text { RBL } \\
141\end{array}$ & 25 & 18 & 0 & 0 & 0.00 & 0 & $\begin{array}{l}\text { Absence of Seed Set } \\
\text { and Abscission of } \\
\text { Crossed Flowers }\end{array}$ \\
\hline 31 & $\begin{array}{l}\text { SML } 1455 \times \\
\quad \text { RBL } 1\end{array}$ & 40 & 32 & 8 & 4 & 12.50 & 55.55 & $\begin{array}{l}\text { Viable Dimpled } \\
\text { Seeds }\end{array}$ \\
\hline 32 & $\begin{array}{l}\text { SML } 1455 \times \\
\quad \text { RBL } 6\end{array}$ & 40 & 34 & 6 & 3 & 8.82 & 28.57 & $\begin{array}{l}\text { Tiny, Dimpled } \\
\text { Seeds }\end{array}$ \\
\hline 33 & $\begin{array}{l}\text { SML } 1455 \times \\
\text { RBL } 9\end{array}$ & 55 & 41 & 11 & 5 & 12.19 & 47.37 & $\begin{array}{l}\text { Viable Dimpled } \\
\text { Seeds }\end{array}$ \\
\hline 34 & $\begin{array}{l}\text { SML } 1455 \times \\
\quad \text { RBL } 33\end{array}$ & 28 & 21 & 0 & 0 & 0.00 & 0 & $\begin{array}{c}\text { Absence of Seed Set } \\
\text { and Abscission of } \\
\text { Crossed Flowers }\end{array}$ \\
\hline 35 & $\begin{array}{l}\text { SML } 1455 \times \\
\text { RBL } 140\end{array}$ & 24 & 16 & 2 & 0 & 0.00 & 0 & $\begin{array}{c}\text { Abscission of young } \\
\text { Fruits }\end{array}$ \\
\hline 36 & $\begin{array}{l}\text { SML } 1455 \times \\
\text { RBL } 141\end{array}$ & 28 & 21 & 2 & 0 & 0.00 & 0 & $\begin{array}{l}\text { Abscission of Young } \\
\text { Fruits }\end{array}$ \\
\hline \multicolumn{9}{|c|}{ Vigna Mungo $\times$ Vigna Umbellata } \\
\hline 37 & $\begin{array}{l}\text { Mash } 338 \times \\
\quad \text { RBL } 1\end{array}$ & 60 & 41 & 9 & 5 & 11.11 & 60.00 & $\begin{array}{l}\text { Viable Dimpled } \\
\text { Seeds }\end{array}$ \\
\hline 38 & $\begin{array}{l}\text { Mash } 338 \times \\
\quad \text { RBL } 6\end{array}$ & 30 & 18 & 5 & 1 & 5.55 & 40.00 & $\begin{array}{l}\text { Tiny, Dimpled } \\
\text { Seeds }\end{array}$ \\
\hline
\end{tabular}




\begin{tabular}{|c|c|c|c|c|c|c|c|c|}
\hline 39 & $\begin{array}{l}\text { Mash } 338 \times \\
\quad \text { RBL } 9\end{array}$ & 60 & 44 & 14 & 5 & 11.36 & 50.00 & $\begin{array}{l}\text { Viable Dimpled } \\
\text { Seeds }\end{array}$ \\
\hline 40 & $\begin{array}{l}\text { Mash } 338 \times \\
\text { RBL } 33\end{array}$ & 44 & 35 & 6 & 2 & 5.71 & 33.33 & $\begin{array}{l}\text { Tiny, Dimpled } \\
\text { Seeds }\end{array}$ \\
\hline 41 & $\begin{array}{l}\text { Mash } 338 \times \\
\text { RBL } 140\end{array}$ & 28 & 18 & 0 & 0 & 0.00 & 0 & $\begin{array}{l}\text { Absence of Seed Set } \\
\text { and Abscission of } \\
\text { Crossed Flowers }\end{array}$ \\
\hline 42 & $\begin{array}{l}\text { Mash } 338 \times \\
\text { RBL } 141\end{array}$ & 42 & 34 & 2 & 2 & 5.88 & 40.00 & $\begin{array}{l}\text { Tiny, Dimpled } \\
\text { Seeds }\end{array}$ \\
\hline 43 & $\begin{array}{l}\text { Mash } 114 \times \\
\quad \text { RBL } 1\end{array}$ & 45 & 38 & 9 & 4 & 10.52 & 20.00 & $\begin{array}{l}\text { Viable Dimpled } \\
\text { Seeds }\end{array}$ \\
\hline 44 & $\begin{array}{l}\text { Mash } 114 \times \\
\quad \text { RBL } 6\end{array}$ & 30 & 21 & 4 & 0 & 0.00 & 0 & $\begin{array}{l}\text { Abscission of Young } \\
\text { Fruits }\end{array}$ \\
\hline 45 & $\begin{array}{l}\text { Mash } 114 \times \\
\text { RBL } 9\end{array}$ & 44 & 37 & 8 & 4 & 10.81 & 55.55 & $\begin{array}{l}\text { Viable Dimpled } \\
\text { Seeds }\end{array}$ \\
\hline 46 & $\begin{array}{l}\text { Mash } 114 \times \\
\text { RBL } 33\end{array}$ & 25 & 18 & 3 & 1 & 5.55 & 25.00 & Tiny, Dimpled Seeds \\
\hline 47 & $\begin{array}{l}\text { Mash } 114 \times \\
\text { RBL } 140\end{array}$ & 46 & 36 & 8 & 3 & 8.33 & 25.00 & Tiny, Dimpled Seeds \\
\hline 48 & $\begin{array}{l}\text { Mash } 114 \times \\
\text { RBL141 }\end{array}$ & 42 & 32 & 6 & 2 & 6.25 & 42.86 & $\begin{array}{l}\text { Viable Dimpled } \\
\text { Seeds }\end{array}$ \\
\hline 49 & $\mathrm{~T} 9 \times \mathrm{RBL} 1$ & 38 & 28 & 6 & 2 & 7.14 & 66.67 & $\begin{array}{l}\text { Tiny, Dimpled } \\
\text { Seeds }\end{array}$ \\
\hline 50 & T $9 \times$ RBL 6 & 52 & 42 & 9 & 3 & 7.14 & 20.00 & $\begin{array}{l}\text { Tiny, Dimpled } \\
\text { Seeds }\end{array}$ \\
\hline 51 & T $9 \times$ RBL 9 & 34 & 22 & 4 & 1 & 4.54 & 25.00 & $\begin{array}{l}\text { Viable Dimpled } \\
\text { Seeds }\end{array}$ \\
\hline 52 & T $9 \times$ RBL 33 & 58 & 48 & 7 & 4 & 8.33 & 20.00 & $\begin{array}{l}\text { Tiny, Dimpled } \\
\text { Seeds }\end{array}$ \\
\hline 53 & T $9 \times$ RBL 140 & 32 & 18 & 3 & 0 & 0.00 & 0 & $\begin{array}{c}\text { Absence of Seed Set } \\
\text { and Abscission of } \\
\text { Crossed Flowers }\end{array}$ \\
\hline 54 & T $9 \times$ RBL 141 & 25 & 18 & 2 & 0 & 0.00 & 0 & $\begin{array}{l}\text { Abscission of Young } \\
\text { Fruits }\end{array}$ \\
\hline 55 & $\begin{array}{l}\text { Shekhar } 2 \times \\
\text { RBL } 1\end{array}$ & 38 & 24 & 7 & 2 & 8.33 & 54.55 & $\begin{array}{l}\text { Viable Dimpled } \\
\text { Seeds }\end{array}$ \\
\hline 56 & $\begin{array}{l}\text { Shekhar } 2 \times \\
\quad \text { RBL } 6\end{array}$ & 34 & 28 & 6 & 2 & 7.14 & 50 & $\begin{array}{l}\text { Tiny, Dimpled } \\
\text { Seeds }\end{array}$ \\
\hline 57 & $\begin{array}{l}\text { Shekhar } 2 \times \\
\text { RBL } 9\end{array}$ & 24 & 16 & 5 & 0 & 0.00 & 0 & $\begin{array}{l}\text { Abscission of Young } \\
\text { Fruits }\end{array}$ \\
\hline 58 & $\begin{array}{l}\text { Shekhar } 2 \times \\
\text { RBL } 33\end{array}$ & 44 & 36 & 8 & 3 & 8.33 & 19.81 & $\begin{array}{l}\text { Tiny, Dimpled } \\
\text { Seeds }\end{array}$ \\
\hline 59 & $\begin{array}{l}\text { Shekhar } 2 \times \\
\text { RBL } 140\end{array}$ & 34 & 25 & 7 & 3 & 8.50 & 17.98 & $\begin{array}{l}\text { Tiny, Dimpled } \\
\text { Seeds }\end{array}$ \\
\hline 60 & $\begin{array}{l}\text { Shekhar } 2 \times \\
\text { RBL } 141\end{array}$ & 50 & 39 & 11 & 4 & 10.25 & 29.41 & Tiny, Dimpled Seeds \\
\hline 61 & Co $5 \times$ RBL 1 & 32 & 22 & 5 & 0 & 0.00 & 0 & $\begin{array}{l}\text { Abscission of Young } \\
\text { Fruits }\end{array}$ \\
\hline 62 & Co $5 \times$ RBL 6 & 20 & 12 & 3 & 0 & 0.00 & 0 & $\begin{array}{l}\text { Abscission of Young } \\
\text { Fruits }\end{array}$ \\
\hline 63 & Co $5 \times$ RBL 9 & 35 & 26 & 6 & 0 & 0.00 & 0 & $\begin{array}{l}\text { Abscission of Young } \\
\text { Fruits }\end{array}$ \\
\hline 64 & Co $5 \times$ RBL 33 & 24 & 13 & 4 & 1 & 7.69 & 25 & Tiny, Dimpled Seeds \\
\hline 65 & $\begin{array}{c}\text { Co } 5 \times \mathrm{RBL} \\
140\end{array}$ & 20 & 14 & 3 & 1 & 7.14 & 25 & Tiny, Dimpled Seeds \\
\hline 66 & $\begin{array}{c}\text { Co } 5 \times \mathrm{RBL} \\
141\end{array}$ & 25 & 16 & 0 & 0 & 0.00 & 0 & $\begin{array}{l}\text { Absence of Seed Set } \\
\text { and Abscission of } \\
\text { Crossed Flowers }\end{array}$ \\
\hline
\end{tabular}




\begin{tabular}{|c|c|c|c|c|c|c|c|c|}
\hline 67 & $\begin{array}{l}\text { Palampur } 93 \times \\
\text { RBL } 1\end{array}$ & 68 & 55 & 14 & 5 & 9.09 & 33.33 & $\begin{array}{l}\text { Viable Dimpled } \\
\text { Seeds }\end{array}$ \\
\hline 68 & $\begin{array}{l}\text { Palampur } 93 \times \\
\text { RBL } 6\end{array}$ & 35 & 26 & 7 & 1 & 3.84 & 0 & $\begin{array}{l}\text { Viable Dimpled } \\
\text { Seeds }\end{array}$ \\
\hline 69 & $\begin{array}{c}\text { Palampur } 93 \times \\
\text { RBL } 9\end{array}$ & 34 & 25 & 8 & 1 & 4.00 & 33.33 & Viable Good Seeds \\
\hline 70 & $\begin{array}{l}\text { Palampur } 93 \times \\
\text { RBL } 33\end{array}$ & 26 & 19 & 4 & 1 & 5.26 & 25.00 & Tiny, Dimpled Seeds \\
\hline 71 & $\begin{array}{l}\text { Palampur } 93 \times \\
\text { RBL } 140\end{array}$ & 25 & 14 & 0 & 0 & 0.00 & 0 & $\begin{array}{l}\text { Absence of Seed Set } \\
\text { and Abscission of } \\
\text { Crossed Flowers }\end{array}$ \\
\hline 72 & $\begin{array}{l}\text { Palampur } 93 \times \\
\text { RBL } 141\end{array}$ & 24 & 15 & 3 & 0 & 0.00 & 0 & $\begin{array}{c}\text { Abscission of Young } \\
\text { Fruits }\end{array}$ \\
\hline \multicolumn{9}{|c|}{ Vigna Radiata $\times$ Vigna Mungo } \\
\hline 73 & $\begin{array}{l}\text { SML } 1455 \times \\
\text { Mash } 338\end{array}$ & 36 & 30 & 13 & 7 & 23.33 & 76.71 & $\begin{array}{l}\text { Viable Shrivelled } \\
\text { Seeds }\end{array}$ \\
\hline 74 & $\begin{array}{l}\text { SML } 1455 \times \\
\text { Mash } 114\end{array}$ & 37 & 30 & 12 & 6 & 20.00 & 81.8 & $\begin{array}{l}\text { Viable Shrivelled } \\
\text { Seeds }\end{array}$ \\
\hline 75 & $\begin{array}{l}\text { HUM } 12 \times \\
\text { Mash } 338\end{array}$ & 40 & 30 & 17 & 11 & 36.66 & 85.18 & $\begin{array}{l}\text { Viable Shrivelled } \\
\text { Seeds }\end{array}$ \\
\hline 76 & $\begin{array}{l}\text { HUM } 12 \times \\
\text { Mash } 114\end{array}$ & 36 & 28 & 13 & 8 & 28.57 & 73.71 & $\begin{array}{l}\text { Viable Shrivelled } \\
\text { Seeds }\end{array}$ \\
\hline 77 & $\begin{array}{l}\text { ML } 1464 \times \\
\text { Mash } 338\end{array}$ & 50 & 32 & 18 & 12 & 37.50 & 75.00 & $\begin{array}{l}\text { Viable Shrivelled } \\
\text { Seeds }\end{array}$ \\
\hline 78 & $\begin{array}{l}\text { ML } 1464 \times \\
\text { Mash } 114\end{array}$ & 42 & 20 & 13 & 5 & 25.00 & 74.35 & $\begin{array}{l}\text { Viable Shrivelled } \\
\text { Seeds }\end{array}$ \\
\hline 79 & $\begin{array}{l}\text { Pusa } 0672 \times \\
\text { Mash } 338\end{array}$ & 56 & 34 & 15 & 9 & 26.47 & 62.07 & $\begin{array}{l}\text { Viable Shrivelled } \\
\text { Seeds }\end{array}$ \\
\hline 80 & $\begin{array}{l}\text { Pusa } 0672 \times \\
\text { Mash } 114\end{array}$ & 55 & 35 & 17 & 9 & 25.71 & 59.68 & $\begin{array}{l}\text { Viable Shrivelled } \\
\text { Seeds }\end{array}$ \\
\hline
\end{tabular}

Even though crossability barriers were predominant, it was possible to recover interspecific hybrids. The crossability of Vigna radiata $\times$ Vigna umbellata and Vigna radiata $\times$ Vigna mungo was successful only when Vigna radiata was used as female and of Vigna mungo $\times$ Vigna umbellata when Vigna mungo was used as female. The percent crossability among different sets of crosses varied from species to species. The differences in pod setting among different set of crosses might be because of wide variation in their genetic architecture leading to differences in cross compatibility. In $V$. radiata $\times V$. umbellata, best combination recorded was HUM 12 $\times$ RBL 9 and HUM $12 \times$ RBL 9 with the highest pods set percentage viz., $16.27 \%$ and $15.78 \%$ respectively. In $V$. mungo $\times$ V. umbellata, the maximum crossability of $11.36 \%$ was noticed in cross, Mash $338 \times$ RBL 9. Similar crossability success were also reported in $V$. radiata $\times V$. umbellata $(29.63 \%), V$. radiata $\times V$. trilobata $(8.48 \%), V$. radiata $\times$ V. aconitifolia (7.69\%) [13] and in V. radiata $\times V$. trilobata $(10.25 \%)$ [14]. similarly, highest pod set of $40.8 \%$ was observed in V. unguiculata $\times$ V. unguiculata var. spontanea [15].

Further, inter-specific hybrids involving three cultivars of urdbean (PDU-1, Palampur-93 and UG-2018) and six of ricebean (Naini, BRS-1, BRS-2, PRR-1, PRR-9301 and Local) exhibited differential response of crossability involving different genotypes $[16,17]$. The timings of anthesis (between 0500 to 0900 HRS), dehiscence of anthers (10 to 14 hours before anthesis) and receptivity of the stigmas (from the time of anthesis up to 6 to 8 hours after anthesis) were identical for the parental species. The length of style was different in the three species- it was $19 \mathrm{~mm}$ in $V$. umbellata, $23 \mathrm{~mm}$ in V. radiata and $21 \mathrm{~mm}$ in $V$. mungo. There are no external barriers, which prevent cross-pollination between $V$. radiata and $V$. umbellata, and $V$. mungo $\times V$. umbellata, because the timing of anthesis, dehiscence of anthers and receptivity of the stigma are identical for both the parental species. Normal pollen germination in both selfed and cross flowers shows that the stigma does not act as barrier.

Absence of seed set and abscission of crossed flowers within 72 hours from pollination in crosses V. radiata $\times$ V. umbellata (TM 96-2 $\times$ RBL 6; Pusa $0672 \times$ RBL 140; KM $2241 \times$ RBL 6; ML $1464 \times$ RBL 140; ML $1464 \times$ RBL 141; HUM $12 \times$ RBL 141 and SML $1455 \times$ RBL 33) and V. mungo $\times$ V. umbellata (Mash $338 \times$ RBL 140; Co $5 \times \mathrm{RBL}$ 141 and Palampur $93 \times$ RBL 140) demonstrate that the first barrier responsible for complete sterility is the delay in pollen tube entry in to the ovules. This might be expected because of the difference in the length of style of three species. Such barriers are known in many other interspecific crosses as well $[13,18,19]$. In addition, relatively more number of crosses of $V$. radiata $\times V$. umbellata showed high abscission of crossed flowers than $V$. mungo $\times V$. umbellata which further supports that the difference in length of style is responsible for complete sterility.Pre fertilization barriers are absent in the interspecific crosses $V$. umbellata $\times$ V. radiata and V. umbellata $\times V$. mungo as evident from normal pollen tube growth in both selfed and crossed flowers and low abscission rate of crosses flower within 72 hours from pollination. 
However, in $V$. radiata $\times V$. mungo, the highest crossability was visualized in hybrid, ML $1464 \times$ Mash 338 (37.5\%). The relatively high number of pods harvested for $V$. radiata $\times V$. mungo suggests that there were no barriers in crossing of these two species for the parental cultivars used. However, barriers were observed in embryogenesis as both inviable and viable seeds were produced, but completely inviable seeds in the reciprocal cross, $V$. mungo $\times V$. radiata. The reciprocal difference in crossability of $V$. radiata and $V$. mungo suggests interaction between genic and cytoplasmic factors [20], which may be the cause of hybrid embryo degeneration when $V$. mungo is used as the female parent $[2,21]$. The high rate of abscission of young fruits between 3 to 30 days after pollination and low seed set in crosses of $V$. radiata $\times V$. umbellata, $V$. mungo $\times V$. umbellata and $V$. radiata $\times V$. mungo are suggestive for the presence of post fertilization barriers. The failure of endosperm nuclei to divide or the delayed endosperm nuclear divisions is responsible for abortion of embryo and the subsequent abscission of young fruits in the interspecific crosses. The failure of embryo to reach maturity might be the probable cause of the production of shrivelled seeds from these crosses.

These Crossability barriers between the cultigen and its wild relative constitute somatoplastic sterility [22]. Such sterility barriers have been recorded in the interspecific crosses between Phaseolus lunatus $\times$ Phaseolus vulgaris [23]. No differences in pod set between the parental cultivars were found when $\mathrm{V}$. radiata or $V$. mungo were used as the female parent. However, significant differences in numbers of seed set were obtained for the interspecific cross V. radiata $\times V$. umbellata and $V$. mungo $\times V$. umbellata. The difference between the V. umbellata cultivars as the pollen parents was highly significant. Based on the percentage pod set and ovule fertility, out of the six ricebean genotypes used, RBL 1 and RBL 9 showed substantially high percent of crossability and better seed set with different cultivars of mungbean and blackgram suggesting that these two genotypes may be utilized for genetic improvement of these crops.

\section{Conclusion}

Different kinds of pre and post fertilization barriers are responsible for complete sterility to low fertility. Despite this, novel genes and alleles from exotic germplasm and related species must be exploited and accordingly hybridization should be utilized to create a wide genetic variation for breeding programs in the Vigna species. Significant progress has been made in basic techniques of tissue culture and in development of techniques to transfer genes from more distantly related taxa. The application of embryo rescue, ovary and ovule culture, chromosome doubling and induced chromosomal exchanges through tissue culture techniques holds considerable promise for the development of new cultivars incorporating genes from wide species.

\section{Acknowledgement}

We thank the Department of Science and Technology, India, for the funding and supporting the research programme by providing the DST INSPIRE Fellowship to the corresponding author.

\section{References}

1. Karthikeyan A, Sudha M, Senthil N, Pandiyan M, Raveendran M, et al. (2012) Screening and identification of RAPD markers linked to MYMV resistance in mungbean (Vigna radiata (L) Wilczek). Archives of Phytopathology and Plant Protection 45(6): 712-716.

2. Ahn CS, Hartmann RW (1977) Interspecific hybridization among four species of the genus Vigna. In: Proc. First Intl. Mungbean Symposium. Asian vegetable Research and Development Centre. Shanhua Taiwan: pp. 240-246.

3. Chen NC, Parrot JF, Jacobs T, Baker LR, Carlson PS (1977) Interspecific hybridization of food legumes by unconventional methods of plant breeding. In: Proc. First Intl. Mungbean Symposium: Asian Vegetable Research and Development Centre. Shanhnua Taiwan: pp. 247-252.

4. Biswas MR, Dana S (1975) Blackgram $\times$ ricebean cross. Cytologia 40: 787-795.

5. Chen NC, Baker LR, Honma S (1983) Interspecific crossability among four species of Vigna food legumes. Euphytica 32(3): 925-937.

6. Chen HK, Mok MC, Shanmugasundaram S, Mok DWS (1989) Interspecific hybridization between Vigna radiata (L.) Wilczek and V. glabrescens. Theoretical and Applied Genetics 78: 641-647.

7. Boiling M, Sander DA, Matlock RS (1961) Mungbean hybridization technique. Agronomy Journal 53: 54-55.

8. Pandiyan M, Senthil N, Ramamoorthi N, Muthiah AR, Tomooka N, et al. (2010) Interspecific hybridization of Vigna radiata $\times 13$ wild Vigna species for developing MYMV donar. Electronic Journal of Plant Breeding 1: 600-610.

9. Pande K, Raghavanshi SS, Prakesh P (1990) Induced high yielding amphiploid of Vigna radiata $\times$ Vigna mungo. Cytologia 55: 249-253.

10. Subramanian A, Muthiah AR (2000) Studies on incompatibility barriers operating in crosses between Vigna mungo (L.) Hepper and Vigna radiata (L.) Wilczek. Legume Research 24(2): 87-91.

11. Singh DK, Singh MN, Vaishamayan A, Raj P, Singh NK, et al. (2016) Crossability Studies in Interspecific $F_{1}$ Hybrids of Mungbean [Vigna radiata (L) Wilczek and Urdbean [Vigna mungo (L) Hepper]. Advances in Life Sciences 5(5): 1801-1805.

12. Bhanu AN, Singh MN, Srivastava K (2017) Consequence of weather conditions for affecting crossability in three Vigna species. Electronic Journal of Plant Breeding 8(2): 572-576.

13. Bharathi A, Vijay Selvaraj KS, Veerabadhiran P, Subba Lakshmi B (2006) Crossability barriers in mungbean (Vigna radiata L. Wilczek): with its wild relatives. Indian Journal of Crop Science 1(1-2): 120-124.

14. Pandiyan M, Senthil N, Suresh R, Chakravarthy N, Packiraj D, et al. (2012) Inter-specific hybridization of Vigna radiata $\times \mathrm{V}$. trilobata. Wudpecker Journal of Agricultural Research 1(6): 233-234.

15. Nwosu DJ, Aladele S, Adeosun JO, Nwadike C, Awa EN (2013) Cross compatibility and $\mathrm{F}_{1}$ reproductive potential of cultivated cowpea varieties and a wild relative (Subsp. unguiculata var. spontenea). Greener Journal of Agricultural Sciences 3(5): 391-395.

16. Mittal RK, Katna G, Sood BC (2005) Inter-specific hybridization in the genus Vigna. In: Proceedings of Fourth International Food Legume Research. New Delhi, India.

17. Dhiman R, Mittal RK, Chaudhary HK, Yadav AK (2013) Crossability relationship between blackgram (Vigna mungo) and ricebean (V umbellata) for successful blackgram $\times$ ricebean hybridization programme. Indian Journal of Agricultural Sciences 83: 907-911.

18. Maheswari P, Rangaswamy NS (1965) Embryology in relation to physiological and Genetics. In: Perston RD (Edn.). Advances in Botanical Research, Vol 2. Academic Press, London, UK pp. 219-312. 
19. Gopinathan MC, Babu CR, Shivanna KR (1986) Interspecific hybridization between ricebean (Vigna umbellata) and its wild relative (V. minima): Fertility - Sterility Relationships, Euphytica 35(3): 1017-1022

20. Stebbins GL (1958) The inviability weakness and sterility of interspecific hybrids. Advances in Genetics 9: 147-215.

21. Subramanian A, Muthiah AR (2001) Interspecific hybridization between V. radiata (L.) Wilczek and blackgram V. mungo (L.) Hepper. Legume Research 24(3): 154-158.

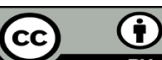

This work is licensed under Creative Commons Attribution 4.0 License

Submission Link: http://biomedres.us/submit-manuscript.php
22. Mayo, Oliver (1980) Theory of Plant Breeding. Clarendon press Oxford.

23. Mok DWS, Mok MC, Rabakorihanta A (1979) Fertilization and early embryo development in reciprocal interspecific crosses of Phaseolus. Theoretical and Applied Genetics 57: 59-65.

$\begin{array}{ll}\text { BIOMEDICAL } & \text { Assets of Publishing with us } \\ \text { RESEARCHES } & \text { - Global archiving of articles } \\ \text { - Immediate, unrestricted online access } & \text { - Rigorous Peer Review Process } \\ & \text { - Authors Retain Copyrights } \\ \end{array}$

